\title{
Calculation of the rotation shells on axisymmetric load taking the creep into account
}

\author{
Anton Chepurnenko ${ }^{1 *}$, Levon Mailyan ${ }^{1}$, Batyr Yazyev $^{1}$ and Akram Ivanov ${ }^{1}$ \\ ${ }^{1}$ Don State Technical University, 344022, Sotcialisticheskaya, 162, Rostov-on-Don, Russia
}

\begin{abstract}
The paper presents a derivation of governing equations and the method of calculation of shells of revolution taking the creep into account. Moment theory is considered. The problem is reduced to a system of two second order differential equations. We also present an example of the calculation of reinforced concrete shell on the effect of its own weight. Viscoelastic model of hereditary concrete aging is used.
\end{abstract}

\section{Introduction}

Axisymmetric problem is one of the important problems of structural mechanics and theory of elasticity. Examples of axially symmetric load acting on the shell of revolution are the dead weight, uniform snow load, the fluid pressure in the tank, and so on.

In this case, resolving equations of the theory of thin shells are greatly simplified. In some cases calculation can be carried out on the membrane theory. However, membrane theory can not account for the edge effect, which occurs in support zone [1]. Calculation of the shells by bending theory is linked with the solution of systems of differential equations with variable coefficients [2-3]. With regard to the calculation based on the creep, currently there are only some partial solutions [4-8].

\section{Derivation of resolving equations}

We consider the rotation shell, which is in terms of axisymmetric stress state. Element produced by cutting the two neighboring meridional planes and two normal sections is shown in Fig. 1.

For this element equilibrium equations can be written as [3]:

$$
\begin{aligned}
& \frac{N_{\varphi}}{R_{1}}+\frac{N_{\theta}}{R_{2}}+\frac{1}{r R_{1}} \frac{d}{d \varphi}(Q r)+p=0 ; \\
& \frac{1}{r R_{1}} \frac{d}{d \varphi}\left[\left(Q \cos \varphi+N_{\varphi} \sin \varphi\right) r\right]=-p_{z} ; \\
& \frac{d}{d \varphi}\left(M_{\varphi} r\right)-Q r R_{1}-M_{\theta} \frac{d r}{d \varphi}=0,
\end{aligned}
$$

*Corresponding author: anton_chepurnenk@mail.ru 
where $p$ - normal component of the surface load, $t$ - tangential component of the external load, $p_{z}=p \cos \varphi+t \sin \varphi-$ the vertical component of the external load.

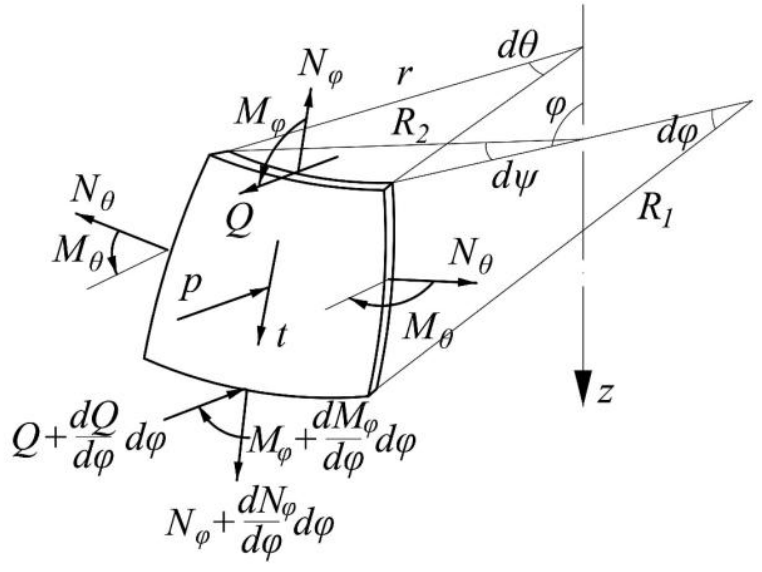

Fig. 1. Equilibrium of rotation shell element

Integrating the second equation in (1) in the range of $\varphi_{0}$ to $\varphi$ we will get:

$$
r\left(N_{\varphi} \sin \varphi+Q \cos \varphi\right)=F(\varphi) \text {, }
$$

where $F(\varphi)=-\int_{\varphi_{0}}^{\varphi} p_{z} R_{1} r d \varphi+C$.

The constant $C$ is equal to 0 if the top edge of the shell (when $\varphi=\varphi_{0}$ ) is not loaded.

The total deformation of the shell is represented as the sum of the middle surface deformation and deformation caused by the change of curvature:

$$
\begin{aligned}
& \varepsilon_{\varphi}=\varepsilon_{\varphi}^{0}+\chi_{\varphi} z ; \\
& \varepsilon_{\theta}=\varepsilon_{\theta}^{0}+\chi_{\theta} z .
\end{aligned}
$$

The deformations of the middle surface are defined as follows:

$$
\begin{aligned}
\varepsilon_{\varphi}^{0} & =\frac{1}{R_{1}} \frac{d v}{d \varphi}-\frac{w}{R_{1}} ; \\
\varepsilon_{\theta}^{0} & =\frac{v \cos \varphi-w \sin \varphi}{r},
\end{aligned}
$$

where $w$ and $v$-displacements respectively in the direction normal to the shell surface and the tangent to the meridian.

Changes of the shell curvature are defined as follows:

$$
\begin{aligned}
& \chi_{\varphi}=-\frac{1}{R_{1}} \frac{d \alpha}{d \varphi} ; \\
& \chi_{\theta}=-\frac{\alpha}{R_{2}} \operatorname{ctg} \varphi
\end{aligned}
$$

where $\alpha=\frac{v}{R_{1}}+\frac{1}{R_{1}} \frac{d w}{d \varphi}-$ the rotation angle of the normal.

Physical equations can be written as: 


$$
\begin{aligned}
& \varepsilon_{\theta}=\frac{1}{E}\left(\sigma_{\theta}-v \sigma_{\varphi}\right)+\varepsilon_{\theta}^{*} \\
& \varepsilon_{\varphi}=\frac{1}{E}\left(\sigma_{\varphi}-v \sigma_{\theta}\right)+\varepsilon_{\varphi}^{*},
\end{aligned}
$$

where $\varepsilon_{\varphi}^{*}, \varepsilon_{\theta}^{*}-$ creep strains.

We express from (6) stresses through the strains:

$$
\begin{aligned}
& \sigma_{\theta}=\frac{E}{1-v^{2}}\left(\varepsilon_{\theta}+v \varepsilon_{\varphi}-\left(\varepsilon_{\theta}^{*}+v \varepsilon_{\varphi}^{*}\right)\right) \\
& \sigma_{\varphi}=\frac{E}{1-v^{2}}\left(\varepsilon_{\varphi}+v \varepsilon_{\theta}-\left(\varepsilon_{\varphi}^{*}+v \varepsilon_{\theta}^{*}\right)\right) .
\end{aligned}
$$

Bending moments are defined as follows:

$$
\begin{gathered}
M_{\theta}=\int_{-\frac{h}{2}}^{\frac{h}{2}} \sigma_{\theta} z d z=\frac{E}{1-v^{2}} \int_{-\frac{h}{2}}^{\frac{h}{2}}\left(\varepsilon_{\theta}^{0}+v \varepsilon_{\varphi}^{0}+z\left(\chi_{\theta}+v \chi_{\varphi}\right)-\left(\varepsilon_{\theta}^{*}+v \varepsilon_{\varphi}^{*}\right)\right) z d z= \\
=D\left(\chi_{\theta}+v \chi_{\varphi}\right)-M_{\theta}^{*}=-D\left(\frac{\alpha}{R_{2}} \operatorname{ctg} \varphi+\frac{v}{R_{1}} \frac{d \alpha}{d \varphi}\right)-M_{\theta}^{*} ; \\
M_{\varphi}=\int_{-\frac{h}{2}}^{\frac{h}{2}} \sigma_{\varphi} z d z=D\left(\chi_{\varphi}+v \chi_{\theta}\right)-M_{\varphi}^{*}=-D\left(\frac{v \alpha}{R_{2}} \operatorname{ctg} \varphi+\frac{1}{R_{1}} \frac{d \alpha}{d \varphi}\right)-M_{\varphi}^{*},
\end{gathered}
$$

where $\quad D=\frac{E h^{3}}{12\left(1-v^{2}\right)} \quad-\quad$ cylindrical rigidity; $\quad M_{\theta}^{*}=\frac{E}{1-v^{2}} \int_{-\frac{h}{2}}^{\frac{h}{2}}\left(\varepsilon_{\theta}^{*}+v \varepsilon_{\varphi}^{*}\right) z d z$ $M_{\varphi}^{*}=\frac{E}{1-v^{2}} \int_{-\frac{h}{2}}^{\frac{h}{2}}\left(\varepsilon_{\varphi}^{*}+v \varepsilon_{\theta}^{*}\right) z d z$.

We introduce new variable $V=R_{2} Q$. Substituting (8) in the last equation (1), we obtain the first governing equation for the functions $\alpha$ and $V$ :

$$
\begin{gathered}
\frac{R_{2}}{R_{1}} \frac{d^{2} \alpha}{d \varphi^{2}}+\frac{d \alpha}{d \varphi}\left[\frac{d}{d \varphi}\left(\frac{R_{2}}{R_{1}}\right)+\frac{R_{2}}{R_{1}} \operatorname{ctg} \varphi\right]+\alpha\left(-\operatorname{ctg}^{2} \varphi \frac{R_{1}}{R_{2}}-v\right)+\frac{R_{1} V}{D}= \\
=\frac{1}{D \sin \varphi}\left[M_{\theta}^{*} R_{1} \cos \varphi-\frac{d}{d \varphi}\left(M_{\varphi}^{*} r\right)\right] .
\end{gathered}
$$

For deriving the equation of compatibility of strains, we differentiate the second equation in (4) by $\varphi$ :

$$
\begin{gathered}
\frac{d \varepsilon_{\theta}^{0}}{d \varphi}=\frac{1}{r}\left(\frac{d v}{d \varphi} \cos \varphi-v \sin \varphi-\frac{d w}{d \varphi} \sin \varphi-w \cos \varphi\right)-\frac{1}{r^{2}} \frac{d r}{d \varphi}(v \cos \varphi-w \sin \varphi)= \\
==\frac{1}{r}\left(\cos \varphi\left(\frac{d v}{d \varphi}-w\right)-\sin \varphi\left(\frac{d w}{d \varphi}+v\right)\right)-\frac{1}{r} \frac{d r}{d \varphi} \varepsilon_{\theta}^{0} .
\end{gathered}
$$

Taking into account that $\frac{d r}{d \varphi}=R_{1} \cos \varphi, \frac{d v}{d \varphi}-w=R_{1} \varepsilon_{\varphi}^{0}, \frac{d w}{d \varphi}+v=R_{1} \alpha$, deformation compatibility equation becomes: 


$$
\frac{R_{2}}{R_{1}} \frac{d \varepsilon_{\theta}^{0}}{d \varphi}=\left(\varepsilon_{\varphi}^{0}-\varepsilon_{\theta}^{0}\right) \operatorname{ctg} \varphi-\alpha .
$$

From the first two equations (1):

On the other hand,

$$
\begin{gathered}
N_{\theta}=-\frac{1}{R_{1}} \frac{d V}{d \varphi}-p R_{2}-\frac{1}{R_{1} \sin ^{2} \varphi} F(\varphi) ; \\
N_{\varphi}=-\frac{\operatorname{ctg} \varphi}{R_{2}} V+\frac{1}{R_{2} \sin ^{2} \varphi} F(\varphi) .
\end{gathered}
$$

$$
\begin{gathered}
N_{\theta}=\int_{-\frac{h}{2}}^{\frac{h}{2}} \sigma_{\theta} d z=\frac{E}{1-v^{2}} \int_{-\frac{h}{2}}^{\frac{h}{2}}\left(\varepsilon_{\theta}^{0}+v \varepsilon_{\varphi}^{0}+z\left(\chi_{\theta}+v \chi_{\varphi}\right)-\left(\varepsilon_{\theta}^{*}+v \varepsilon_{\varphi}^{*}\right)\right) d z=\frac{E h\left(\varepsilon_{\theta}^{0}+v \varepsilon_{\varphi}^{0}\right)}{1-v^{2}}-N_{\theta}^{*} \\
N_{\varphi}=\int_{-\frac{h}{2}}^{\frac{h}{2}} \sigma_{\varphi} d z=\frac{E h}{1-v^{2}}\left(\varepsilon_{\varphi}^{0}+v \varepsilon_{\theta}^{0}\right)-N_{\varphi}^{*},
\end{gathered}
$$

where $N_{\theta}^{*}=\frac{E}{1-v^{2}} \int_{-\frac{h}{2}}^{\frac{h}{2}}\left(\varepsilon_{\theta}^{*}+v \varepsilon_{\varphi}^{*}\right) d z ; \quad N_{\varphi}^{*}=\frac{E}{1-v^{2}} \int_{-\frac{h}{2}}^{\frac{h}{2}}\left(\varepsilon_{\varphi}^{*}+v \varepsilon_{\theta}^{*}\right) z d z$.

We express from (13), the mean surface deformations through the longitudinal forces:

$$
\begin{aligned}
& \varepsilon_{\varphi}^{0}=\frac{1}{E h}\left(N_{\varphi}-v N_{\theta}\right)+\frac{1}{E h}\left(N_{\varphi}^{*}-v N_{\theta}^{*}\right) ; \\
& \varepsilon_{\theta}^{0}=\frac{1}{E h}\left(N_{\theta}-v N_{\varphi}\right)+\frac{1}{E h}\left(N_{\theta}^{*}-v N_{\varphi}^{*}\right) .
\end{aligned}
$$

Substituting the expression for the longitudinal forces (12) in the expression for the deformation of the middle surface (14), and then the middle surface deformation compatibility equation of deformation (11), we obtain the second governing equation:

$$
\frac{R_{2}}{R_{1}} \frac{d^{2} V}{d \varphi^{2}}+\frac{d V}{d \varphi}\left[\frac{d}{d \varphi}\left(\frac{R_{2}}{R_{1}}\right)+\frac{R_{2}}{R_{1}} \operatorname{ctg} \varphi\right]+V\left(\nu-\frac{R_{1}}{R_{2}} \operatorname{ctg}^{2} \varphi\right)=E h \alpha R_{1}+\Phi(\varphi)+\Phi^{*}(\varphi),
$$

where

$$
\Phi(\varphi)=-\frac{d}{d \varphi}\left(p R_{2}^{2}\right)+t R_{2}\left(R_{2}+v R_{1}\right)-\frac{F(\varphi)}{\sin ^{2} \varphi}\left[\left(\frac{R_{1}}{R_{2}}-\frac{R_{2}}{R_{1}}\right) \operatorname{ctg} \varphi+\frac{d}{d \varphi}\left(\frac{R_{2}}{R_{1}}\right)\right]
$$

$\Phi^{*}(\varphi)=R_{2} \frac{d}{d \varphi}\left(N_{\theta}^{*}-v N_{\varphi}^{*}\right)-\operatorname{ctg} \varphi R_{1}(1+v)\left(N_{\varphi}^{*}-N_{\theta}^{*}\right)$.

Thus, the problem of calculation of axially loaded shells of rotation of arbitrary shape is reduced to a system of two differential equations (9) and (15) for the functions $\alpha$ and $V$.

\section{Solution of the problem}

Here is an example calculation of the tank, the middle surface of which is a one-sheeted hyperboloid of rotation (Fig. 2). The equation of the meridian of this surface is as follows:

$$
r=\frac{a}{b} \sqrt{b^{2}+z^{2}},
$$

where $a, b$-parameters of hyperbole. 


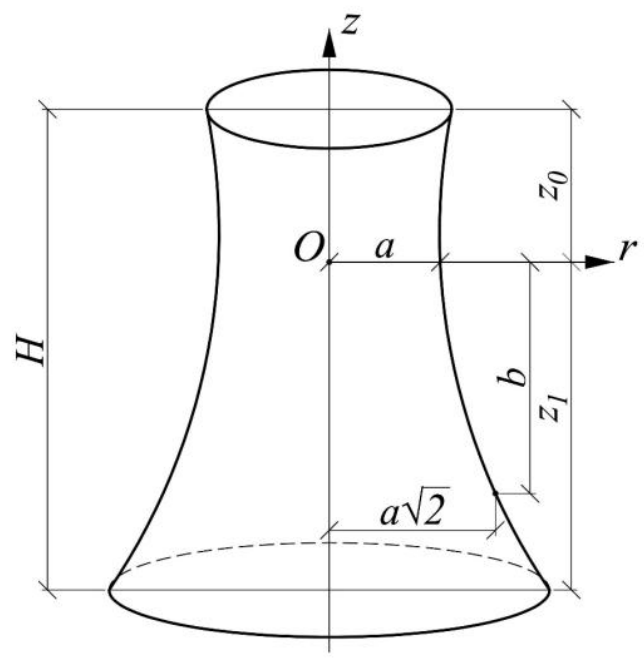

Fig. 2. Hyperboloid of rotation

We performed the calculation of reinforced concrete shell on the effect of its own weight at the following initial data: $a=13 \mathrm{~m}, b=28.16 \mathrm{~m}, z_{0}=-7.8 \mathrm{~m}, z_{1}=45.5 \mathrm{~m}$, $E=2 \cdot 10^{7} \mathrm{kPa}, v=0.17, h=0.25 \mathrm{~m}$, specific gravity of material $\gamma=24 \mathrm{kN} / \mathrm{m}^{3}$. As the creep law equation we used viscoelastic models of hereditary aging [9-12]:

$$
\begin{aligned}
& \varepsilon_{\varphi}(t)=\frac{1}{E(t)}\left(\sigma_{\varphi}(t)-v \sigma_{\theta}(t)\right)-\int_{\tau_{0}}^{t}\left\{\sigma_{\varphi}(\tau)-v \sigma_{\theta}(\tau)\right\} \frac{\partial C(t, \tau)}{\partial \tau} d \tau ; \\
& \varepsilon_{\theta}(t)=\frac{1}{E(t)}\left(\sigma_{\theta}(t)-v \sigma_{\varphi}(t)\right)-\int_{\tau_{0}}^{t}\left\{\sigma_{\theta}(\tau)-v \sigma_{\varphi}(\tau)\right\} \frac{\partial C(t, \tau)}{\partial \tau} d \tau,
\end{aligned}
$$

where $C(t, \tau)$ - creep measure, which is written as follows:

$$
C(t, \tau)=C \frac{e^{\alpha t}-e^{\alpha \tau}}{e^{\alpha t}-1}+B\left(e^{-\gamma \tau}-e^{-\gamma t}\right) .
$$

The transition from the integral form of creep equations to the differential form is shown in [13]. Method for determining creep strains is given in the works [14-17]. Rheological constants of concrete in the calculation were taken to be: $\gamma=0.062$ day $^{-1}, \mathrm{C}=$ $3.77 \cdot 10^{-8} \mathrm{kPa}^{-1}, \mathrm{~B}=5.68 \cdot 10^{-8} \mathrm{kPa}^{-1}, \alpha=0.032$ day ${ }^{-1}$. Start time $\tau_{0}=28$ days. We considered hinged at the base shell.

\section{Results and discussion}

Diagram of changes of the maximum value of the deflection $w$ is shown in Fig. 3. The greatest value of the deflection at $t \rightarrow \infty$, and $\mathrm{t}=\tau_{0}$ differ in 1.95 times. Redistribution of internal forces during creep was not observed. The difference between the highest values of the bending moments $M \varphi$ at $t=\tau_{0}$ and $t \rightarrow \infty$ is only $1.29 \%$.

\section{Summary}

Obtained equations allow calculation of the shell of rotation of arbitrary shape at any load and an arbitrary law of the connection between stresses and creep strains. The 
calculation of a concrete shell in the form of hyperboloid showed that concrete creep does not have a significant impact on the magnitude of internal forces.

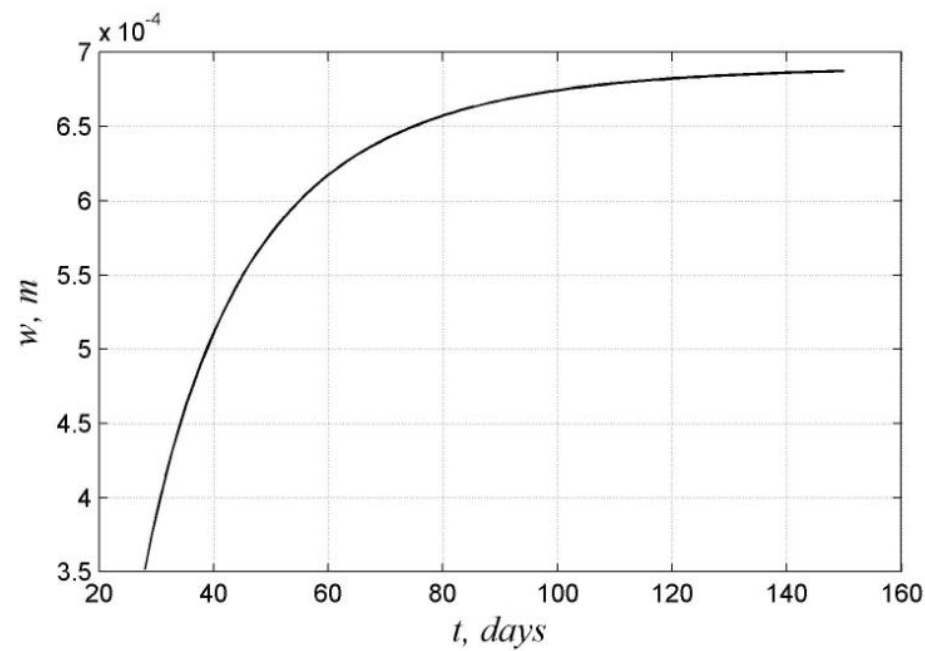

Fig. 3. Diagram of deflection growth

\section{References}

1. A.L. Gol'Denveizer. Theory of Elastic Thin Shells: Solid and Structural Mechanics. Vol. 2. Elsevier, 2014.

2. Benson, D. J., et al. A large deformation, rotation-free, isogeometric shell. Computer Methods in Applied Mechanics and Engineering 200.13 (2011): 1367-1378.

3. A. Tomás, P. Martí. Shape and size optimisation of concrete shells. Engineering Structures 32.6 (2010): 1650-1658.

4. G. Gerard. A critical strain approach to creep buckling of plates and shells. Journal of the Aerospace Sciences (2012).

5. H. Altenbach, K. Naumenko. Shear correction factors in creep-damage analysis of beams, plates and shells." JSME International Journal Series A Solid Mechanics and Material Engineering 45.1 (2002): 77-83.

6. J. Bockhold, Y. S. Petryna. Creep influence on buckling resistance of reinforced concrete shells. Computers \& structures 86.7 (2008): 702-713.

7. A. E. Gemma The creep deformation of symmetrically loaded circular cylindrical shells. Journal of the Aerospace Sciences (2012).

8. J. T. Boyle, J. Spence. Stress analysis for creep. Elsevier, 2013.

9. A.G. Tamrazyan, The mechanics of concrete creep: monograph (Moscow, 2012)

10. Diab, Hesham, and Zhishen Wu. A linear viscoelastic model for interfacial long-term behavior of FRP-concrete interface. Composites Part B: Engineering 39.4 (2008): 722 730.

11. Creus, Guillermo J. Viscoelasticity-basic theory and applications to concrete structures. Vol. 16. Springer Science \& Business Media, 2012. 
12. Hattel, J. H., and Jesper Thorborg. A numerical model for predicting the thermomechanical conditions during hydration of early-age concrete. Applied Mathematical Modelling 27.1 (2003): 1-26.

13. L.R. Mailyan, A.S. Chepurnenko, A. Ivanov, Calculation of prestressed concrete cylinder considering creep of concrete, Procedia Engineering, 165 (2016). pp. 18531857

14. V.I. Andreev, A.S. Chepurnenko, B.M. Yazyev. Energy method in the calculation stability of compressed polymer rods considering creep, Advanced Materials Research, 1004-1005 (2014). pp. 257-260.

15. V.I. Andreev, B.M. Yazyev, A.S. Chepurnenko, On the Bending of a Thin Plate at Nonlinear Creep, Advanced Materials Research, 900 (2014). pp. 707-710.

16. A.S. Chepurnenko, A.V. Saibel, B.M. Yazyev, Determination of the Breaking Load for Concrete Slabs Based on the Deformation Theory of Plasticity, Procedia Engineering, 150 (2016). pp. 1694-1700.

17. A.S. Chepurnenko, B.M. Yazyev, A.A. Savchenko, Calculation for the Circular Plate on Creep Considering Geometric Nonlinearity, Procedia Engineering, 150 (2016) 